\title{
IMPORTANCIA DEL MERCADO SECUNDARIO INTERNACIONAL DE TÍTULOS DE LA DEUDA EXTERNA: SU CONTRIBUCIÓN A LAS FINANZAS INTERNACIONALES
}

\author{
IMPORTANCE OF SECONDARY MARKET INTERNATIONAL FOREIGN DEBT \\ SECURITIES: THEIR CONTRIBUTION TO INTERNATIONAL FINANCE
}

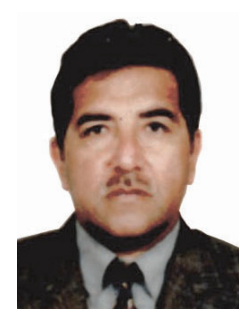

Ricardo Norberto Villamonte Blas*

Docente Asociado de la Facultad de Ciencias Económicas

Universidad Nacional Mayor de San Marcos-UNMSM / Lima-Perú

[Recepción: Julio de 2013/ Conformidad: Agosto 2013]

\section{RESUMEN}

La mejor contribución que académicamente se le puede brindar a acontecimientos financieros que contribuyeron al desarrollo de las finanzas internacionales es la de estudiarlos a profundidad de manera que, sus tratamientos y resultados permitan a los especialistas e interesados tener una mejor comprensión de su evolución, características e importancia. El Mercado Secundario Internacional de Títulos de la Deuda Externa (MSDE) se le tipifica como un mercado muy singular, definiéndolo como un mercado de negociación bilateral con características especiales, que ha tenido una participación crucial. Si bien su contribución fue marginal respecto al importe total de la deuda de ese entonces se convirtieron 59 mil millones de dólares frente a los 600 mil millones de dólares de deuda, gracias a él. En primer lugar, se constituyen nuevas modalidades financieras que bajo el mecanismo de mercado, permitieron aliviar o solucionar parcialmente la difícil situación de los estados financieros de sus principales actores y en segundo lugar, permitieron esclarecer y reconocer a los acreedores que el valor de mercado era la base para establecer una negociación que se constituyera en una importante contribución para la solución parcial tanto para ellos como para los deudores de la deuda externa la que llegó a ser considerada como un producto financiero obsoleto.

\section{PALABRAS CLAVE:}

Mercado secundario internacional de títulos de la deuda externa, mercado de negociación bilateral, nuevas modalidades financieras, valor de mercado, producto financiero obsoleto, deuda impagable.

\begin{abstract}
The best contribution academically can provide financial events that contributed to the development of international finance is to study them in depth so that their treatments and outcomes allow experts and stakeholders to have a better understanding of its evolution, characteristics and importance. The International Securities Secondary Market of External Debt (MSDE) is typified as a very unique market, defining it as a bilateral trading market with special features, which has had a crucial role. Although its contribution was marginal compared to the total amount of debt then 59 billion dollars were converted versus 600 billion dollars in debt, thanks to this market. First, they constitute new financial arrangements that under the market mechanism, allowed to alleviate or partially solve the plight of the financial statements of its principal and secondary actors, allowed to clarify and recognize the creditors that the market value was the basis for a negotiation that would serve as an important contribution to the partial solution for them and for borrowers of foreign debt which came to be regarded as an outdated financial product.
\end{abstract}

\section{KEYWORDS:}

International secondary market for external debt, bilateral trading market, new financial arrangements, market value, obsolete financial product, unpayable debt.

* Doctor en Ciencia Económicas y Empresariales-Universidad Autónoma de Madrid. España. Email: rivillamonteb@hotmail.com 


\section{INTRODUCCIÓN}

En el periodo 1973-1982, el endeudamiento externo de los países en desarrollo creció a niveles muy comprometedores y de muy difícil administración. En 1982, la capacidad de pago de los países deudores estaba seriamente disminuida y comprometida como consecuencia de factores externos a ellos (1). Entre dichos factores se encuentran la política de ajuste económico de Estados Unidos y la implementación de su nueva Ley de Comercio Exterior, que afectaban negativamente a los precios de las exportaciones de los países deudores. Paralelamente a ello, otro factor gravitante fue el hecho de que la política monetaria de ese país determinó una política de tasa de interés y una sobre valoración del dólar, que reducían notablemente la capacidad de pago de los países deudores.

A diciembre de 1982, casi el 60\% (2) de la deuda externa pública de América Latina provenía de bancos privados internacionales. Estas instituciones habían superado ampliamente la participación de los proveedores, organismos financieros multilaterales y bilaterales. Las condiciones financieras de sus préstamos eran más exigentes en términos de tasas de interés, plazos, garantías y otros elementos colaterales al crédito. Dichos préstamos alcanzaron a financiar hasta el $60 \%$ del déficit en cuenta corriente de la balanza de pagos, en el período 1968- 1981. La tasa PRIME RATE real que en el periodo 1970 - 1979 no superó el $2 \%$, en el periodo 1981- 1984 se mantuvo en un rango entre el $7 \%$ y el $8 \%$; es decir, creció en promedio en un 400\%. Los bancos concedieron el $99.8 \%$ de sus préstamos bajo condiciones duras), las que en su máximo nivel de dureza equivalían a otorgar préstamos de una tasa mayor al $12 \%$, para préstamos de 1 a 5 años.

El servicio de la deuda externa llegó en los años 1978 y 1982 a 57.2 y 135.9 miles de millones de dólares respectivamente; es decir, creció del 17\% al $23 \%$ del total de la deuda externa, la carga financiera alcanzada era de difícil atención.

Esta deuda generada posteriormente fue tipificada como "producto financiero obsoleto" pues por las condiciones crediticias en que se pactaron, así como la coyuntura económica y el comportamiento y proyección de las principales variables macroeconómicas, que para muchos especialistas era prácticamente impagable.

Las diferentes explicaciones y opiniones de cómo se había llegado a esa difícil situación, partía del reconocimiento de que sus diferentes actores eran de una u otra manera, responsables de haber llegado a esa situación y que para la solución de la misma, debía partirse de ese reconocimiento y que el planteamiento de solución no solo se precise en las variables financieras involucradas a través de refinanciaciones y restructuraciones de la deuda, sino que también, en otros elementos propios del desarrollo económico y social internacional. Los expertos coincidían que de no encontrarse un consenso en los acuerdos del tratamiento de la deuda externa, no tendría una solución sostenible.

Los países deudores eran responsables por cuanto se perdió la objetividad en los requerimientos de nuevos préstamos, cada vez eran menos préstamos que para la importación de alimentos y la realización de proyectos productivos que refuercen el crecimiento y su capacidad de pago, cada vez se pedían más préstamos para proyectos de larga maduración, gastos de defensa y principalmente, financiamiento del déficit público como para atender el servicio de la deuda.

La banca acreedora también era responsable por cuanto en una coyuntura de recesión mundial, orientaron el reciclaje de los petrodólares a los países pobres que dependían de los países grandes y de la participación proteccionista, de éstos últimos en el mercado mundial, actuaron como empresas comercializadoras. Conocían que las recuperaciones de sus préstamos con tasas de interés que crecieron hasta un $400 \%$ de su valor promedio en esas condiciones eran de muy difícil cumplimiento.

Durante 1982 y 1983 en los organismos regionales como el Sistema Económico Latinoamericano (SELA) y la Comisión Económica para América Latina ( CEPAL ) se declaró que el deterioro de los términos de intercambio, el proteccionismo y la alta tasa de interés se constituían en factores que los países de la región no podían controlary se solicitó 
un esfuerzo de ajuste equitativo y coordinado entre todos los actores involucrados. Esos planteamientos no tuvieron acogida por parte de los países acreedores.

Los países deudores mostraban su preocupación en atender el servicio de la deuda, las negociaciones de restructuración de la deuda externa no prosperaron, fueron rechazadas por los acreedores. La deuda había llegado a un nivel inmanejable, por lo que en ese mismo año, se empieza a gestar una filosofía latinoamericana para enmarcar una nueva política necesaria para las renegociaciones: el enfoque de la corresponsabilidad.

Los académicos de los países desarrollados manifestaron que los grandes déficits de sus economías, así como, sus políticas monetarias restrictivas generaron altas tasas de interés que afectaban al mundo. La Iglesia solicitaba que los países desarrollados apliquen planes de cooperación y asistencia para los países endeudados (3).

El enfoque de la corresponsabilidad no prosperó se trataba de un enfoque financiero y económico a la vez, debido a la falta de liquidez como de la posible insolvencia de los deudores que eran partes de un mismo problema y donde todos los actores deberían participar activamente para su solución. Ante ese entorno los bancos acreedores empezaron a rematar sus títulos o pagarés de la deuda externa por debajo de su valor nominal, se iba constituyendo así un mercado financiero internacional informal al que se le denomina: Mercado Secundario Internacional de Títulos de la Deuda Externa (MSDE).

\section{JUSTIFICACIÓN DEL CRECIMIENTO Y PROLIFERACIÓN DE OPERACIONES EN EL MSDE}

Ante la pérdida de confianza de cobrar sus acreencias(4) al inicio, los principales acreedores: los bancos comerciales internacionales fueron los que empezaron con las primeras operaciones en el mercado, a través de remates directos de sus acreencias de deuda debido a la necesidad coyuntural de liquidez y a la exposición de su capital. A falta de recursos lí- quidos y ante las reducidas posibilidades de recuperar los fondos prestados, algunos bancos prefirieron deshacerse de sus títulos de deuda, asumiendo pérdidas por los descuentos. En esta instancia se empiezan a generar las primeras operaciones de remates de la deuda externa en el MSDE, las cotizaciones de los títulos de la deuda externa empiezan a decaer.

Luego, al tomarse conocimiento de que los países en desarrollo insistían y justificaban que la deuda era impagable y que reclamaban una corresponsabilidad compartida entre ellos y los acreedores para iniciar un nuevo tratamiento a las renegociaciones y reestructuraciones de la deuda, los bancos comerciales aceleraron sus remates de deuda, en esta situación el volumen de operaciones de la deuda externa en el MSDE empieza a crecer y su cotización a bajar más rápidamente. En esta etapa los bancos acreedores empiezan a incorporan nuevas modalidades al remate de deuda externa como por ejemplo :el intercambio de deudas de diferentes países en monedas similares o diferentes con o sin similitud de los plazos, tasas y otras condiciones en que se pactaron los prestamos; swaps o canjes de títulos de la deuda por valores equivalentes o no, a cambio de acciones, bonos u otros activos financieros, bajo diferentes perfiles de rendimientos, riesgos, plazos, etc. En muchos casos los bancos acreedores se iban alejando lentamente de los montos originales de sus acreencias. En suma el mercado era ahora más útil por cuanto en él, se empezaba a diversificar operaciones que permitía aliviar los niveles de exposición del capital de los bancos acreedores y perfilar su deuda hacia posiciones más favorables para su gestión bancaria(5).

Posteriormente, los propios países deudores conocedores que continuaba hacia la baja la cotización de su deuda externa consideraron conveniente participar en él, en un primer momento a través de compras directas de sus propias deudas, posteriormente cuando toman conocimiento que de su propia deuda externa se podían generar nuevos negocios a través de su conversión en inversión participan de una manera más activa ofertando nuevas modalidades de conversión (6). 
La banca acreedora norteamericana (principal acreedor con el $60 \%$ ) respecto a bancos similares de otros países, se encontraban en una situación de desventaja al no gozar de sistemas de provisiones de deudas flexibles con ágiles deducciones fiscales que le permitiesen digerir más la deuda. Más aun, cuando se oficializaron los menú de opciones de conversión de deuda externa en inversión de los países deudores, los cuales se constituían en un gran negocio y permitían que los bancos acreedores cambiaran su rol de prestamistas a inversores, los bancos norteamericanos consideraron conveniente solicitar la flexibilización de la Regulación "K" a la Reserva Federal por cuanto dicha norma limitaba su participación como inversores en el extranjero. Ante estas limitaciones, y ante la presión de los bancos acreedores, mediante la enmienda de febrero de 1987, la Junta de Gobernadores de la "Federal Reserve Board" "flexibilizó" la Regulación "K" a fin de apoyarlos en su gestión y en su participación en la transformación de la deuda externa de los países en desarrollo en inversión (7). Esta acción permitió mejorar notablemente la disposición de los bancos para participar más en el mercado MSDE pero ahora, a ellos se le añadía la participación de los especuladores y en general, de inversionistas que deseaban participar en la conversión, así en 1988 el volumen de operaciones de conversión de deuda llega a su pico más alto. El mercado entraba a su etapa de proliferación, en él se establecen diferentes tipos de operaciones de conversión de deuda (8), entre ellas las siguientes:

"Debt-Products Swaps": pago de exportaciones entregando títulos de deuda externa; "Debt-Service Swaps": pago de servicios de empresas públicas y privadas con deuda externa; "Debt-Capital RiskSwaps": cambio de deuda externa por capital de riesgo; "Debt-Cash Swaps": conversiones de títulos de deuda por activos finncieros de liquidez reconocida y poder cancelatorio inmediato, inclusive divisas; "Debt-Equity-Swaps": cambio de deuda externa por acciones representativas de una nueva inversión y “Debt-Local Money Swaps":compra de deuda externa con moneda nacional; "Debt -Local Debt Swaps”: permutas que permitían cancelaciones de deuda local entregando a cambio pagarés de la deuda externa, se buscaba contribuir al saneamiento de deudas internas; "Debt - Off Shore Swaps": Son capitalizaciones de deuda externa mediante compañías off-shore, para ejecutar inversiones en los países deudores; "Debt-Exit Bonds": conversiones generalmente por bonos de salida emitidos por los gobiernos de los países deudores; "Debt- Junk Bonds": bonos que se obtienen por la conversión de títulos de deuda externa pero que tiene alto riesgo y alto rendimiento; "Debt- Multilateral Bonds Swaps": conversiones de títulos de deuda por bonos emitidos y garantizados por organismos multilaterales mediante la apertura de fondos en fideicomiso que le permitan recomprar deuda a un país; "Debt-Nature-Swaps": conversión de títulos por divisas o fondos locales para financiar proyectos de desarrollo o mejoramiento del medio ambiente en los países deudores.

Mediante esta etapa ante un "desierto financiero y de inversiones" originado por la crisis de la deuda externa de 1982, se logra la apertura de una "nueva frontera de negociación”. Esta situación representa la constitución de almácigos e incubadoras de nuevos negocios en pro de la solución de la deuda externa. Después que se flexibiliza la Regulación “K”, el Mercado de Debt Swaps llega a su máximo nivel de expansión en 1988: 22,392 millones de dólares.

A los bancos también les interesaba canjear deudas en el marco de la conversión, por cuanto en adición a la necesidad coyuntural de liquidez, ante las reducidas posibilidades de recuperar los fondos prestados y de deshacerse de sus títulos de deuda aún asumiendo pérdidas por los descuentos, la conversión les permitía: equilibrar el riesgo de sus carteras mediante la diversificación, cambiar pagarés de deuda externa por activos financieros de mayor rentabilidad y menor riesgo; obtener deducciones fiscales por la venta de deuda morosa; reducir los costos de supervisión y administración de la cartera morosa; reducir el riesgo país y constituir reservas especiales.

Pero es importante destacar que las modalidades de convertir su deuda en acciones "Debt-EquitySwaps" y, "Debt-Capital Risk-Swaps" lo acreditaban como dueños de un nuevo proyecto empresarial 
que les permitía cambiar su posición "de acreedor de un préstamo" a "accionista de una inversión" posición en la cual aquel importe que sacrificaba como producto del descuento o remate de su deuda por debajo del valor nominal: el valor de mercado, que era la base para fijar un nuevo negocio, podía posteriormente ser ampliamente recuperado mediante la generación de las utilidades de los productos o servicios de los nuevos proyectos.

\section{ESTRATEGIAS DE LA CONVERSIÓN DE DEUDA EXTERNA DE LOS PAÍSES ENDEUDADOS}

Se conceptúa a los Debt-Swaps como aquella gama de productos financieros diversificado "no estandarizado" que se diversifican a partir de un producto financiero básico: la deuda externa. La connotación de "no estandarizado" se da por el reconocimiento de que las "condiciones- financieras" de cada operación, no son estables, estas se establecen en cada operación en una negociación entre el acreedor de la deuda y el gobierno deudor, ambos tratan de sacar ventaja de su posición en la determinación de las variables de negociación de cada proyecto de conversión de la deuda.

Los países deudores establecieron programas de conversión a los que también se les llamó "menú de opciones", de un análisis conjunto de esos programas se puede resumir que los objetivos generales de los gobiernos deudores en la implementación de la conversión fueron:

a) Atender y reducir el servicio y lo principal de la deuda.

b) Incrementar los niveles de inversión extranjera y nacional.

c) Contribuir al saneamiento financiero de empresas: financieras y no financieras tanto nacionales como extranjeras establecidas en el país.

d) Desarrollar proyectos empresariales que por su cuantía y riesgo, difícilmente podían ser asumidos por el gobierno o inversionistas privados nacionales.
Conocidos los objetivos generales, el problema radicaba en que los recursos de cada nación podían ofertarse en la conversión y cuál era la mejor ventaja de su aplicación (9). Ello obligó a que se determine una estrategia en la selección y aplicación de dichos recursos, asíse estableció que los debt swaps deberían:

-Utilizarse para el aprovechamiento de la capacidad instalada, ociosa o sub-utilizada del país.

- Requerir una inversión adicional mínima del gobierno.

- Representar una inversión incremental del acreedor.

- Alcanzar al más amplio grupo bancario acreedor, pero bajo una discriminación de acreencias o deudas que les resultara financieramente más favorable. Es decir, se trata de seleccionar entre deuda de largo o corto plazo y, considerando las condiciones financieras en que fueron contraídas.

- Utilizarse en el saneamiento financiero y/o capitalización de empresas financieras y no financieras, en donde ni el sector público ni el sector privado tuviesen la posibilidad en el corto y mediano plazo de efectuar las inversiones necesarias.

- Ofertarse para proyectos nuevos de empresas productivas, inviables por la falta de inversión nacional.

- Utilizarse para la cancelación de adeudos de empresas multinacionales o nacionales con el sistema financiero nacional, a fin de cuidar la salud del sistema financiero nacional.

- Utilizarse como medio de repatriación de capitales.

- Utilizarse para la redenominación a moneda nacional de la deuda internacional y cubrirse del riesgo de cambio.

\section{TIPIFICACIÓN DEL MSDE}

Expuesto los resultados de la conversión queda la interrogante de conocer las características del Mercado Secundario Internacional de Títulos de la Deuda Externa (MSDE) que generó las operaciones antes señaladas. 
El Mercado Secundario de Pagarés de deuda Externa, por sus características no califica dentro de las definiciones tradicionales de los mercados, pero se puede tipificarlo a partir de las características operativas más importantes que se utilizaron, según Shiguiyama (11) se le define como:

\section{Mercado de "monopolio bilateral"}

Por cuanto el bien a negociar es la deuda externa en la que participa su dueño exclusivo o monopolista: el banco y el país deudor o monopsonista, único comprador (nos estamos refiriendo a la primera y principal forma de acercamiento en el mercado, ya que también se dieron otras cuando aparecen inversionistas o especuladores como ofertantes o demandantes), ambos desean maximizar su utilidad.

El monopsonista a partir del valor de mercado ofertaba una tasa de descuento que lo aleje del valor nominal de la deuda, tratando así de fijar "su" valor real de la deuda. El monopolista dejando de lado el valor de mercado que él mismo ocasionó por su remate, buscaba de lograr un precio entre la tasa de descuento ofertada por el deudor y el valor nominal original de su acreencia. La determinación del precio no se daba dentro del periodo normal del mercado bursátil tomaba mucho más tiempo. Sea cual fuere el resultado, el "cierre" de la valoración final no se daba a conocer oficialmente de manera inmediata. El precio de la negociación se establecía "caso por caso".

\section{Mercado sin transparencia}

En este mercado no hay un precio o cotización fija de referencia por cuanto no se llevaba una cotización oficial para cada tipo de deuda. En cada operación respecto al tipo de deuda transada no se registraba en el mercado: la fecha de emisión de la deuda original, el concepto del préstamo internacional original, el importe de la deuda pagada o por pagar, las etapas de renegociación, de reestructuración, entre otras variables a considerar.

\section{Mercado con información privada- Forma- ción de precios}

La información que se impartía al público, la brindaban empresas o consultores expertos, que tomaban conocimiento de los cierres de los negocios.
La información no se registraba bajo la modalidad que empleaban los mercados bursátiles; por lo, tanto no penetraba fidedignamente al mercado, no se brindaba un proceso de legitimización de los precios (12). Estos, servían solamente como una referencia para otros participantes. El mercado no recogía el efecto sobre las cotizaciones de transacciones no concretadas.

\section{Mercado no regulado}

Las evaluaciones realizadas permiten indicar que el mercado no estaba regulado; es decir, no había un mercado o bolsa central a través de la cual se oficialicen públicamente los precios, como tampoco las normas para su funcionamiento. No existía un lugar central donde se haya fijado explícitamente las reglas del juego para las transacciones. Esa falta de organización se refleja en el hecho de que muchos accionistas lo conceptúen como un mercado informal, en el sentido de que no existían cotizaciones "oficiales". Las cotizaciones disponibles no garantizaban que se podía obtener títulos de deuda a un precio determinado.

\section{Mercado regulado "de fuera hacia adentro" de forma amplia y diferenciada}

Debe señalarse en esta fase del estudio, que si bien se negociaron 58,669 millones de dólares de deuda, dicho monto pudo ser mayor, de no ser por las regulaciones que se daban desde fuera del mercado y que por lo tanto, controlaban o restringían su expansión. La participación de los bancos ofertantes dependía del tratamiento contable, provisiones para reservas, deducciones fiscales y regulaciones de los sistemas financieros bancarios de cada país, en consecuencia la regulación interna era reemplazada por regulación externa. Debiéndose destacar que la participación mayoritaria fue la de la banca norteamericana, principal acreedor con el $60 \%$ y que fue posterior al resto de bancos que tenían normas y regulaciones que hacían viable su participación en inversiones en el exterior, cuando se dio la flexibilización de la regulación $\mathrm{K}$.

Debe señalarse que el mercado fue afectado por las declaraciones oficiales de los organismos financieros internacionales cuando éstos se pronuncian 
a favor de esquemas de reducción de deuda a través del mecanismo de mercado, ello era entendido por los bancos como cambios que alentaban su participación en el mercado.

\section{Mercado marginal}

Una de las características principales de este mercado es su marginalidad respecto al volumen total de la deuda externa de los países latinoamericanos. En efecto, en este mercado si bien se llegaron a efectuar en términos acumulados para el período 1984-1991: 179,500 operaciones por un total de 59 mil millones de dólares, esta llegó a representar hasta el $6 \%$ de la deuda externa total.

\section{IMPORTANCIA Y CONCLUSIONES DEL MSDE AL DESARROLLO DE LAS FINANZAS INTERNACIONALES}

Son tres las grandes contribuciones de este mercado:

En primer lugar, en este mercado se gestaron diferentes modalidades financieras que permitieron aliviar, atenuar o solucionar parcialmente las difíciles situaciones financieras en las que se encontraban los principales actores de la deuda externa: los bancos acreedores y los gobiernos deudores .

En segundo lugar, este mercado ha tenido una participación importante, se convirtieron $59 \mathrm{mil}$ millones de dólares de deuda .

En tercer lugar, los bancos acreedores quienes en un primer momento fueron adversos a las convocatorias y acuerdos del Sistema Económico Latinoamericano (SELA) y de la Comisión Económica para América Latina (CEPAL) para que las renegociaciones se diesen bajo el enfoque de corresponsabilidad compartida, reconocieron indirectamente su responsabilidad cuando participaron en las conversiones de deuda tomando como base de negociación el "valor de mercado", que se constituyó en la base para establecer un nuevo ámbito de negociación y en una importante contribución para la solución parcial, tanto para ellos, como para los deudores de la deuda externa, la que llegó a ser considerada como un producto financiero obsoleto por cuanto los términos en que se negociaron, en sus 10 últimos años, determinaban que era una deuda impagable.

\section{NOTAS:}

(1) La dimensión de la deuda y sus efectos negativos son tratados en el estudio de: DEVLIN, Robert: "Banca Internacional y el endeudamiento de América Latina”, en: Comercio Exterior MX 33 (7), Julio de 1983; y también en DOOLEY, M. "Análisis de las posiciones de las deudas externas en ocho países en desarrollo", en: Monetaria MX7 (4) Oct - Dic. 1984.

(2) Las estadísticas y los efectos negativos del financiamiento externo son analizados y han sido tomados de: KISIC , Drago: “De la Corresponsabilidad a la Moratoria, el caso de la deuda externa peruana, 1970-1986", Fundación Ebert Lima - Perú, 1987.

(3) Los principales documentos que se pueden revisar son los siguientes : la "Declaración Conjunta de Quito en Enero de 1984"; "Las bases para un entendimiento de la Conferencia Especial Especializada Extraordinaria sobre Financiamiento Externo", "Al Servicio de la Comunidad Humana: un informe ético de la Cuestión de la Deuda Internacional", en: KISIC, Draqo, Op. Cit.

(4) Varios autores analizaron la cuestionada capacidad de pago, entre ellos podemos señalar a: GUTH William, "Banca Internacional: Nueva Fase, en: Boletín CEMLA MX 28 (3) Mayo - Junio 1982. PONIACHEK, H. "Banca Internacional y el Mercado Latinoamericano”, en: Administración de Empresas AR 15(170), Mayo 1984. También se encuentra una exposición detallada sobre la pérdida de confianza de la banca acreedora en: KRUGMAN, Paul: "Economía Internacional: Teoría y Política”, Mc Graw Hill, Madrid, 1994. Capítulos 18 y 21.

(5) Un mayor tratamiento de los instrumentos teóricos asociados a los mecanismos de gestión y digestión de la deuda externa pueden apreciarse en: PARK, Y. y ZUICK, J. ."International Banking in Theory and Practice". Addison, Wes- 
ley, 1985; también en COOPER, J. “Management and Regulation of Banks”, New York, St. Martin Press, 1984.; y en: DALI, Richard: “The Regulation of International Banking”, Cambridge, Woodhead-Faulkner Ltd., 1984.

(6) Se tomó como fuentes para este análisis los estudios de:

VELASCO A. y LARRAIN F. “Los Intercambios Swaps de Deuda”, en: Trimestre Económico MX 57 (225) Enero - Marzo 1990.

LANEY L. "Mercado Secundario de Deuda de los Países en Desarrollo: Observaciones y repercusiones en las políticas", en: Boletín CEMLA MX 34 (2),Tr. de Economic Review (Banco de la reserva Federal de Dallas) (US) Julio, 1987.

(7) Debe considerarse que el Sistema Financiero de los Estados Unidos de Norteamérica era uno de los "más regulados en el mundo”. Al respecto véase:

KIM, S.H.y MILLER, S. “Competitive Structure of the International Banking Industry”, Lexinqthon, Health, 1983.

Board of Governors of the Federal Reserve System: a)Regulation "K”; "International Banking Operations”, 12-CFF., October 1735 y su Enmienda de Febrero de 1987; b) Regulation "Y”: "Sank Holding Companies and Changes in Banking Control"; 12-CFR-Z25, March 1989.

EL COMERCIO, "Reserva Federal de EE.UU.: Liberaliza Conversión de Deuda Externa en Capital”. Lima - Perú, 24 de Febrero de 1987;

EYSELL, Thomas; FRASER, Donald; RANGAN, Nanda: "Debt Equity Swaps, Regulation K, and Bank returns", en: Journal of Banking and Finance No 13, North Holland, 1989.

(8) El autor para la determinación de las características que se señalan, consultó y analizó las siguientes fuentes: FRENCH, Martin. "Variations on a theme of Swaps", en: Euromoney, London, Julio de 1987.y BERGSMAM, Joel y EDISXS, Wayne. "Debt Equity Swaps and Foreign Direct Investment in Latin America”, en: Debt Equity Swaps in the 1990's; Swaps under the Brady Umbrella. Rubin Steve, Washington D.C., The Economist Publications, 1989.

(9) También se tomó como fuentes para este análisis los estudios de:

VELASCO A. y LARRAIN F. “Los Intercambios Swaps de Deuda”, Op. Cit. y de LANEY LEROY: "Mercado Secundario de Deuda de los Países en Desarrollo: Observaciones y repercusiones en las políticas”, Op. Cit.

(10) Se consideraron a los siguientes autores en los temas referidos a la Diversificación Instrumental o Institucional Financiera: EITEMAN, D. y STONEHILL, A.: "Multinational Business Finance”. Addison - Wesley Publishing Co., Los Angeles, 1979y : KIDWELL, David S., y PETERSON, Richard L. "Financial Institutions, Markets and Money", Saunders College Publishing, New York, 1983.

(11) SHIGUIYAMA, David y VASQUEZ, José: “El Mercado Secundario de Pagarés de Deuda Externa”, en : GARRIDO LECCA: Deuda Externa del Problema a la Posibilidad. Universidad del Pacífico, Julio de 1993.

(12) Suratgar es el autor que más ha analizado las características en que se informaba sobre la formación de precios. Véase al respecto: SURATGAR, D. "DebtEquityConversions: a Guide for Decisión Makers", United Nations Center on Transnational Corporations, United Nations, New York, 1990. 


\section{REFERENCIAS BIBLIOGRÁFICAS}

1. BERGSMAM, Joel y EDISXS, Wayne. "Debt Equity Swaps and Foreign Direct Investment in Latin America”, en: Debt Equity Swaps in the 1990's; Swaps under the Brady Umbrella. Rubin Steve, Washington D.C., The Economist Publications, Washington D.C., 1989.

2. Board of Governors of the Federal Reserve System.

3. COOPER, J. "Management and Regulation of Banks”, New York, St. Martin Press, 1984.

4. DALI, Richard. "The Regulation of International Banking”, Cambridge, Woodhead-Faulkner Ltd., 1984.

5. DEVLIN, Robert. Banca Internacional y el endeudamiento de América Latina. En: Comercio Exterior MX 33 (7), Julio de 1983.

6. DOOLEY, M. "Análisis de las posiciones de las deudas externas en ocho países en desarrollo", en: Monetaria MX 7 (4) Oct - Dic. 1984.

7. EITEMAN, D. y STONEHILL, A. "Multinational Business Finance”. Addison - Wesley Publishing Co., Los Angeles, 1979.

8. EL COMERCIO, "Reserva Federal de EE.UU.” Liberaliza Conversión de Deuda Externa en Capital. Lima - Perú, Febrero de 1987.

9. EYSELL, Thomas; FRASER, Donald; RANGAN, Nanda. "Debt Equity Swaps, Regulation K, and Bank Stocks Returns", en: Journal of Banking and Finance No 13, North Holland, 1989.

10. FRENCH, Martin. "Variations on a theme of Swaps”, en: Euromoney, London, Julio de 1987.

11. GUTH, William. "Banca Internacional": Nueva Fase, en: Boletín CEMLA MX 28 (3) Mayo Junio 1982.

12. KIDWELL, David S., y PETERSON, Richard L. "Financial Institutions, Markets and Money", Saunders College Publishing, New York, 1983.
13. KIM S.H. y MILLER, S. "Competitive Structure of the International Banking Industry”, Lexinqthon, Health, 1983.

14. KISIC, DRAGO. "De la Corresponsabilidad a la Moratoria, el caso de la deuda externa peruana, 1970-1986", Fundación Ebert. Lima - Perú, 1987.

15. KRUGMAN, Paul. "Economía Internacional; Teoría y Política”. Mc Graw Hill, Madrid, 1994.

16. La "Declaración Conjunta de Quito en Enero de 1984; Las bases para un entendimiento de la Conferencia Especial Especializada Extraordinaria sobre Financiamiento Externo- CIES- OEA Caracas, 1983, Al Servicio de la Comunidad Humana: un informe ético de la Cuestión de la Deuda Internacional, en: KISIC, Draqo. Op. Cit.

17. LANEY, Leroy. "Mercado secundario de deuda de los países en desarrollo: Observaciones y repercusiones en las políticas", Boletín CEMLA MX 34(2):69-81 mar.-abr- 1988.

18. LANEY, Leroy. "Mercado secundario de deuda de los países en desarrollo: Observaciones y repercusiones en las políticas. Boletín CEMLA MX 34 (2) Tr. De Economic Review (Banco de la Reserva Federal de Dallas) (US) Julio, 1987.

19. PARK, Y. y ZUICK, 3. "International Banking in Theory and Practice", Addison, Wesley, 1985.

20. PONIACHEK, H. "Banca Internacional y el Mercado Latinoamericano” En: Administración de Empresas AR 15(170), Mayo 1984.

21. Regulation "K"; "International Banking Operations", 12-CFF:,October 1985 y su Enmienda de Febrero de 1987.

22. Regulation " $Y$ ": "Bank Holding Companies and Changes in Banking Control”; 12-CFR-Z25, March 1989. 
23. SHIGUIYAMA, David y VASQUEZ, José. "El Mercado Secundario de Pagarés de Deuda Externa" En: GARRIDO LECCA: Deuda Externa del Problema a la Posibilidad, Universidad del Pacífico, Lima Perú, Julio de 1993.

24. SURATGAR, David. "Debt Equity Conversions: a Guide for Decision Makers”, United Nations
Center on Transnational Corporations, ST/ CTC/ 104,United Nations, New York, 1990.

25. VELASCO, A.; LARRAIN, F. "Macroeconomía básica de los intercambios (Swaps)de deuda”, Trimestre Económico MX 57 (225), enero marzo, 1990. 